\title{
Disfagia en un adolescente como manifestación de la Esofagitis Eosinofílica
}

\author{
Ma Pilar Orgaz Gallego $^{a}$, Esperanza Solera Martínez ${ }^{\mathrm{b}}$, Miguel Ángel Tricio Armero $^{\mathrm{b}}$
}

a Especialista en Medicina

Familiar y Comunitaria, Centro de Salud de Tarancón CEDT (Cuenca).

${ }^{\mathrm{b}}$ Diplomado/a Universitario/a en Enfermería, Centro de Salud de Tarancón CEDT (Cuenca).

Correspondencia: Ma Pilar Orgaz Gallego, Centro de Salud de Tarancón CEDT, C/ Camino Corral de Almaguer s/n, 16400 - Tarancón, Cuenca, España. Correo electrónico: mporgaz@sescam.jccm.es.

Recibido el 26 de agosto de 2011.

Aceptado para su publicación el 2 de septiembre de 2011.

\begin{abstract}
RESUMEN
La Esofagitis Eosinofílica (EE) es una entidad rara, aunque emergente, que debemos sospechar en niños y jóvenes con impactación del bolo alimenticio o disfagia persistente y enfermedad atópica, o en casos de reflujo gastroesofágico refractario a la terapia. El diagnóstico precisa la realización de endoscopia digestiva alta con biopsias y el hallazgo histológico de más de 20 eosinófilos por campo de gran aumento.

Es una inflamación crónica del esófago con una prevalencia en aumento, que requiere un diagnóstico y tratamiento precoces y un seguimiento en el tiempo, pues por el momento se desconocen las consecuencias a largo plazo. Presentamos el caso de un adolescente con disfagia e impactación alimentaria de años de evolución.
\end{abstract}

Palabras clave. Esofagitis Eosinofílica, Trastornos de Deglución.

\section{ABSTRACT}

Eosinophilic Esophagitis causing dysphagia in an adolescent.

Eosinophilic esophagitis (EE) is a rare, but emerging entity that we must consider in children and young patients with food impaction or persistent dysphagia and atopic disease, or in cases of refractory gastroesophageal reflux disease. Diagnosis must be made by mucosal biopsies of the esophagus obtained by esophagogastroduodenoscopy and the histological finding of at least 20 eosinophils per high-power field.

This disease is a chronic inflammation of the esophagus with increasing prevalence, requiring early diagnosis and treatment with consistent follow-up, since long-term consequences are currently unknown. We present the case of an adolescent with dysphagia and food impaction of many years' duration.

Key words: Eosinophilic esophagitis, Deglutition Disorders.

\section{INTRODUCCIÓN}

La esofagitis eosinofílica (EE), también denominada esofagitis alérgica ${ }^{1}$ (1981) es un trastorno inflamatorio crónico de origen desconocido que cursa con disfagia recurrente y episodios de impactación alimentaria esofágica, con una gran variabilidad en la intensidad, frecuencia y duración de los ataques. Esta entidad clínico-patológica, de reciente descripción, se caracteriza por una densa infiltración de granulocitos eosinófilos, limitada exclusivamente a la mucosa esofágica² ${ }^{2}$.

Estamos asistiendo a un incremento de los casos diagnosticados y comunicados (según Fox et al. ${ }^{3}, 24$ hasta 1998 y 200 en 2002) y, aunque se desconoce su incidencia, podría estar en aumento ${ }^{4,5}$. Se podría pensar que es una entidad rara, aunque los últimos estudios hablan de prevalencias de hasta 23 casos $/ 100.000$ habitantes ${ }^{6}$ y muchos autores advierten que se trata de una enfermedad emergente ${ }^{7}$.

Afecta predominantemente a varones jóvenes durante la tercera y cuarta décadas de la vida, que asocian en gran porcentaje manifestaciones atópicas, lo que se ha relacionado con la probable etiología inmunoalérgica del proceso $\mathrm{O}^{4}$, aunque los mecanismos fisiopatológicos de la EE no son del todo conocidos ${ }^{7}$. 
Varios autores coinciden en que es un proceso inflamatorio de etiología inmunoalérgica, determinado por una posible reacción de hipersensibilidad frente a componentes de la dieta o a aeroalergenos ${ }^{7}$. Esta teoría se sustenta en el hecho de que muchos pacientes tienen historia personal y familiar de asma, rinitis alérgica, dermatitis atópica, alergia a fármacos, eosinofilia sanguínea o valores séricos de lgE elevados ${ }^{5,7}$.

Aunque la infiltración eosinofílica primaria del esófago podría deberse a una respuesta de la inmunidad celular, la inmunidad humoral dependiente de los linfocitos $B$ y de la IgE tiene un importante papel en el inicio o el mantenimiento del infiltrado. Los eosinófilos tienen un rol relevante en el desarrollo de la disfagia asociada a la EE y los mastocitos, en las proximidades de las fibras nerviosas aferentes del músculo liso del esófago, contribuyen a la dismotilidad digestiva en estos pacientes ${ }^{7}$.

\section{OBSERVACIONES CLÍNICAS}

Presentamos a un varón de 21 años sin antecedentes patológicos de interés. No alergias conocidas, ni intervenciones quirúrgicas previas. En los antecedentes familiares destaca: madre con alergia a polen de gramíneas, polvo doméstico y sensibilización a sprays (lacas, perfumes, insecticidas etc.).

Hace 5 años consultó porque desde los 12-13 años presentaba clínica de "disfagia alta" para sólidos, inicialmente con la ingesta de arroz y posteriormente con otros alimentos. En el estudio gastroduodenal (febrero 2007) se vio una imagen sugestiva de hernia hiatal por deslizamiento, con reflujo en decúbito, y estómago elongado hipocinético con pliegues y curvaturas regulares sin signos de patología ulcerosa ni tumoral. También se observó evacuación lenta a través de un píloro normal sobre un bulbo de morfología normal y sin signos de patología ulcerosa. La manometría esofágica (abril 2008) mostró disfunción de ambos esfínteres, relajación incompleta del EES, compatible con acalasia cricofaríngea, y relajación incompleta del $\mathrm{EEl}$, inespecífica.

En mayo de 2008 se le realizó una gastroscopia con premedicación y, al visualizar por encima del cricofaríngeo una mucosa de aspecto granujiento, fue derivado a Otorrinolaringología, donde tras fibrolaringoscopia informaron de la existencia de hipertrofia de la pared faríngea posterior y amígdalas linguales como únicos hallazgos. Los hallazgos endoscópicos fueron:

- A nivel esofágico: prácticamente todo el esófago, pero sobre todo el medio e inferior, con "aspecto anillado"; en el tercio superior (a $25 \mathrm{~cm}$ de la arcada dentaria) pequeña imagen pseudodiverticular en la cara lateral derecha; pequeño desgarro en la unión esófagogástrica por las arcadas durante la exploración y pequeña hernia de hiato por deslizamiento de $2 \mathrm{~cm}$. Se tomaron biopsias para descartar EE.

- A nivel gástrico: restos alimentarios sólidos retenidos en cámara gástrica sin lesión obstructiva; leve gastritis crónica de mucosa astral y píloro centrado sin lesiones. Se tomó biopsia de antro.

- A nivel duodenal: bulbo duodenal con restos alimenticios sólidos; mucosa visualizada normal; en la segunda porción duodenal se apreciaron pliegues de tamaño normal, pero con una mucosa ligeramente deshidratada, de aspecto inespecífico. Se tomó biopsia.

Anatomía patológica (mayo 2008): las biopsias de mucosa duodenal presentaban cambios mínimos inespecíficos; las de antro, congestión con ligero aumento del infiltrado inflamatorio crónico en la lámina propia con presencia de eosinófilos en escaso número. En esófago medio se encontró un epitelio escamoso no queratinizado con infiltración por abundantes eosinófilos con tendencia a formar pequeños grupos.

En 2009 se le realizaron pruebas de alergia (IgE total $60,7 \mathrm{KU} / \mathrm{L} ; \mathrm{N}: 0-120$ ) e IgE específica a atún, avellana, bacalao, cebada (alimento), lenteja, manzana, merluza, patata, salmón, trigo (alimento) y arroz, siendo todas negativas $(<0,1 \mathrm{kUI} / \mathrm{L})$. En febrero de 2010 el hemograma no mostró eosinofilia y en noviembre de 2010 muy ligera: $0,9 \mathrm{mil} / \mathrm{mmc}$ (N: 0-0.6).

El paciente fue diagnosticado de acalasia cricofaríngea, EE y hernia hiatal por deslizamiento con reflujo. Se le trató con omeprazol y fluticasona con mejoría clínica al disminuir el número de episodios de atragantamiento (1-2/mes). El paciente con el tiempo abandonó el tratamiento con omeprazol por intolerancia y dejó de utilizar fluticasona.

En Diciembre de 2010 consultó de nuevo por episodios de atragantamiento cada vez más frecuentes y de mayor duración, precisando de ciertas maniobras como la ingesta abundante de 
líquido y/o la flexión anterior del cuello para conseguir el paso del bolo alimenticio, pero también de la provocación de arcadas para lograr la expulsión del contenido alimenticio detenido. La deglución era a veces ruidosa y refería hipersalivación, aunque no presentaba disnea, tos, halitosis, mal sabor de boca, pirosis ni alteración del ritmo intestinal.

Ante la reagudización clínica con aumento de la frecuencia y duración de los episodios, se remitió de nuevo a Digestivo, iniciándose tratamiento con exclusión de alimentos (cereales, huevos, leche, legumbres, arroz, pescado/mariscos, carne de ternera, judías verdes, maíz, frutos secos y embutidos, salvo jamón serrano, y reintroducción paulatina de éstos en la dieta con controles endoscópicos periódicos. Hasta el momento actual, los cereales y la leche (figura 1) son los únicos que han producido una respuesta inflamatoria esofágica, tolerando bien el huevo y el arroz.

\section{COMENTARIOS}

La EE puede presentarse en todas las etnias, aunque parece más frecuente en la raza blanca $y$ en el sexo masculino, tanto en niños como en adultos. En la infancia ocurren más del $65 \%$ de los casos, siendo la edad media del diagnóstico los 10 años $^{5}$. En adultos se describe con mayor frecuencia entre la $3^{a}$ y $5^{a}$ décadas de la vida ${ }^{7}$.
Fox et al. ${ }^{3}$ observaron diferencia en la presentación clínica entre niños y adultos (tabla1). En los niños $<2$ años es más frecuente observar rechazo del alimento y retraso del crecimiento, mientras que los de 3-12 años suelen presentar vómitos, regurgitación, náuseas y dolor torácico. En los $>12$ años la disfagia y la impactación de alimentos son las manifestaciones más habituales, como en el caso descrito. La impactación del bolo alimenticio es la que más frecuentemente conduce al diagnóstico de la EE en pacientes adultos y ocurre en más del $20 \%$ de los casos infantiles de la enfermedad.

El diagnóstico de la EE requiere endoscopia más biopsias y manometría esofágica. La endoscopia puede ser normal hasta en el $9 \%$ de los casos o presentar anomalías $(95 \%)^{5}$, como estenosis regulares concéntricas 0 anillos segmentarios simultáneos que impiden el avance del endoscopio y la visualización de la luz distal. Recuerdan al aspecto de la tráquea. Por ello, este patrón, que es el más frecuente, se conoce como traquealización del esófago, esófago anillado ó esófago felino. Esos anillos corresponden a contracciones intermitentes de la musculatura circular esofágica y son la causa de las impactaciones. Otros hallazgos más sutiles son surcos longitudinales lineales "corrugación esofágica" y exudados blanquecinos puntiformes o papulares, que corresponden a depósitos mucosos ó microabscesos constituidos por eosinófilos ${ }^{5}$.

Adultos

Disfagia

Impactación del alimento

Vómitos

Dolor torácico

Niños

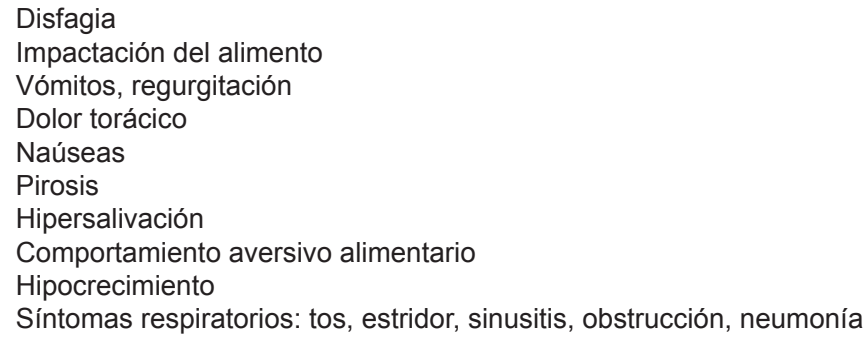

Tabla 1. Sintomas asociados con la EE. Modificado de Fox et $\mathrm{al}^{3}$. 


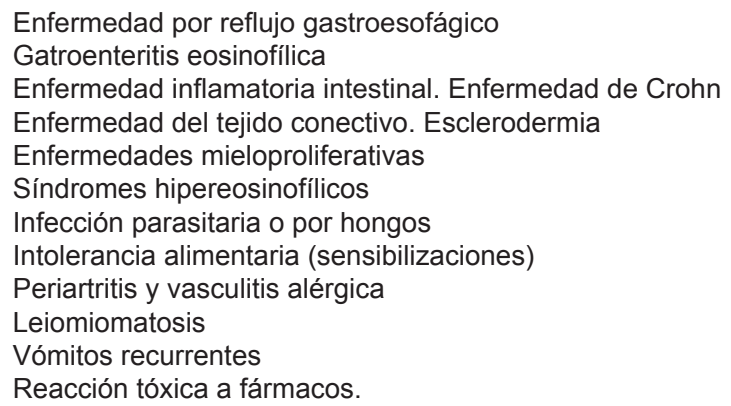

Tabla 2. Diagnóstico diferencial de la EE.

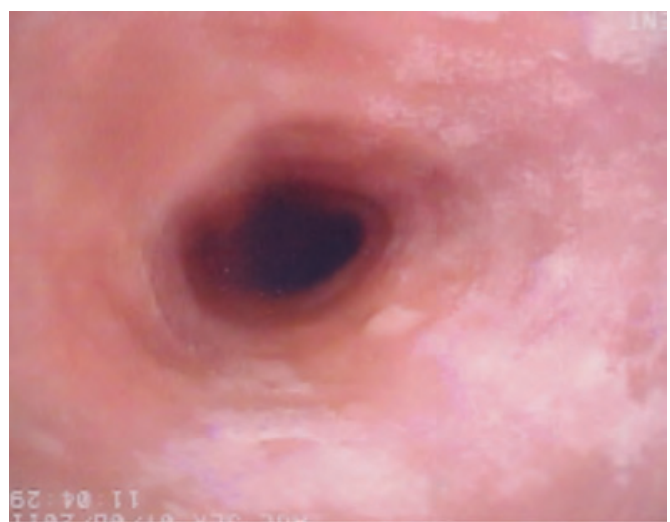

Figura 1. Esofagitis eosinofílica. Anillos esofágicos y exudados blanquecinos papulares (microabscesos formados por eosinófilos).

La endoscopia se acompañará siempre de biopsias en los tres tercios del esófago, aun en ausencia de lesiones, ya que entonces el único criterio diagnóstico para la EE es el anatomopatológico ${ }^{4,7}$. Se recomiendan 5 biopsias, tanto del esófago proximal como distal, pues la sensibilidad diagnóstica es del $55 \%$ con una, llegando al $100 \%$ con las cinco ${ }^{8}$. También se deben tomar de estómago y duodeno para descartar otras enfermedades como gastroenteritis eosinofílica y enfermedad inflamatoria intestinal entre otras.

La presencia de un denso infiltrado de la mucosa esofágica, epitelio peripapilar y lámina propia de los tercios superior y medio del esófago, por más de 20 eosinófilos por campo de mayor aumento, es el criterio histopatológico diferencial ${ }^{7}$, permitiendo el diagnóstico diferencial con la enfermedad por reflujo gastroesofágico "ERGE", en la que el aumento de eosinófilos se produce en capas más superficiales del epitelio y en el tercio distal del esófago, no llegando a los 20 eosinófilos por campo de gran aumento ${ }^{5}$. No existe correlación entre el número de eosinófilos infiltrantes y la gravedad del daño inflamatorio endoscópico ${ }^{7}$.

Respecto a la manometría esofágica, son pocos los casos publicados con estudio de la función motora del esófago, pero en el $65 \%$ de la serie global se han detectado alteraciones motoras, de tipo espástico la mayoría o con hipercontractilidad, cumpliendo criterios de acalasia vigorosa, espasmo esofágico difuso, ondas peristáticas de gran amplitud, etc. En una amplia proporción de pacientes la motilidad esofágica es normal ${ }^{7}$.

El diagnóstico de alimentos y aeroalergenos, que incluye el prick test cutáneo para reacciones de hipersensibilidad tipo I mediadas por IgE y el patch test cutáneo para reacciones de hipersensibilidad tipo IV mediadas por Th2 (linfocitos T helper), han demostrado utilidad para la identificación de sustancias que causarían la EE en ciertos sujetos, permitiendo el éxito terapéutico en la mayoría de casos $^{8}$. Para el diagnóstico diferencial de la EE, ver tabla 2. 
Se ha demostrado que la eliminación de antígenos alimentarios se acompaña de la mejoría clínica e histológica de la EE en la mayoría de los pacientes. Existen 3 formas de eliminarlos:

a) Dieta elemental (gold estándar): reemplazo de todos los alimentos sólidos por una fórmula elemental, nutricionalmente completa, en la que la fuente proteica sean exclusivamente aminoácidos sintéticos. Es efectiva en el $98 \%$ de los niños y adolescentes, pero mal tolerada por su mal sabor, lo que obliga a su administración por sonda nasogástrica o tubo de gastrostomía y tiene un elevado coste ${ }^{9}$.

b) Dieta de eliminación estándar (DESA): se basa en eliminar de la dieta los 6 alimentos que más comúnmente causan $\mathrm{EE}$, como la leche, soja, huevos, trigo, maní/nueces y pescados/ mariscos ${ }^{9}$. Otros también implicados son: patata, pollo, cacahuete y maíz $z^{5}$. Esta dieta permite tomar alimentos sólidos mejorando la adherencia terapéutica, pero su desventaja es el riesgo nutricional del paciente ${ }^{9}$.

c) Dieta de eliminación específica: elimina alimentos específicos (alergenos) basándose en las pruebas alérgicas cutáneas, prick test, y del parche, patch test. Deben vigilarse estrechamente por el riesgo de déficit nutricional ${ }^{5}$. Una vez identificados los alergenos, se administra una dieta libre de ellos, reintroduciéndolos progresivamente en la dieta cada 6 días, evaluando la sintomatología ${ }^{8}$. Hay que tener muy en cuenta el impacto psicológico de este proceso en el paciente y su familia ${ }^{9}$.

Los corticoides consiguen la remisión del infiltrado esofágico, lo cual se asocia a mejoría sintomática. Se recomienda:

- Metilprednisolona a dosis de 0,5 mg/kg/día durante 6 meses con pauta descendente 4 .

- Propionato de Fluticasona a dosis de 440$880 \mathrm{mcg} / \mathrm{día}$ en niños y $880-1.760 \mathrm{mcg} / \mathrm{día}$ en adultos, repartidos en 2-4 dosis, pidiéndole al paciente que tras la aplicación lo degluta para conseguir efecto terapéutico tópico, con resultados similares a los corticoides sistémicos, pero sin los efectos adversos de éstos. Se considera el fármaco a usar como primera línea ${ }^{4,5,8,10}$.

- Budesonida oral: hay casos descritos con buena evolución clínica, endoscópica y anatomopatológica ${ }^{5}$.

En cuanto a otros tratamientos:

- Mepolizumab. Son anticuerpos IgG humanizados anti interleucina-5 que consiguen reducir el número de eosinófilos en sangre y esófago.

- Montelukast. Son inhibidores de los receptores D4 de los leucotrienos que a dosis de 30$40 \mathrm{mg} /$ día parecen tener cierta eficacia. Son defendidos por diferentes autores para evitar los posibles efectos adversos de los esteroides ${ }^{4,10}$. Con montelukast y con los estabilizadores de membrana de los mastocitos, como el cromoglicato, se carece de la suficiente experiencia ${ }^{7}$.

- Las dilataciones endoscópicas proporcionan alivio temporal de los síntomas y tienen mayor tasa de complicaciones graves (desgarros y perforaciones esofágicas), por lo que sólo se utilizarán en casos muy excepcionales, como estrechez esofágica secundaria a estenosis que cause impactación del alimento ${ }^{8}$.

- Los antiácidos Se utilizarán si hay síntomas, ya que un $\mathrm{pH}$ menos ácido favorece la menor activación de los mastocitos, los cuales producen mediadores que a su vez aumentan la actividad de los eosinófilos. Por otra parte, se ha sugerido que el bloqueo ácido impediría la digestión adecuada de péptidos alergénicos, aumentando la sensibilización y consecuentemente la enfermedad alérgica ${ }^{5}$. Una buena respuesta a los inhibidores de la bomba de protones "IBP" no corresponde a la EE, sino a ERGE. La recomendación es evitar los IBP como terapia inicial en pacientes con EE.

El curso de la EE es recurrente ó crónico, con frecuentes recaídas, aunque no parece disminuir la expectativa de vida de quienes la padecen. Sin el tratamiento adecuado, la inflamación persistente de la mucosa esofágica podría conllevar el desarrollo de metaplasia (esófago de Barret, adenocarcinoma de la zona afectada). Ello obliga a un seguimiento cercano de estos pacientes, al estudio y control de posibles complicaciones y a la administración de terapéuticas que aseguren un control eficaz de la enfermedad. Son por tanto necesarios estudios prospectivos a largo plazo.

Puesto que el médico de familia es quien atiende en primera instancia a estos pacientes, deberá tener siempre en mente la posibilidad diagnóstica de la "esofagitis eosinofílica" ante un adolescente o varón joven con clínica de disfagia persistente y/o impactaciones del bolo alimenticio, especialmente si tiene historia personal o familiar de atopia (presente hasta en el $60 \%$ de los adultos), planteándose el principal diagnóstico diferencial con la ERGE. La descripción de nuevos casos ayudará al mejor 
conocimiento de la enfermedad, siendo necesarios estudios prospectivos controlados que permitan comparar la eficacia y seguridad de los diversos tratamientos. Mientras tanto, no se puede establecer un protocolo de actuación adecuado, aunque los corticoides tópicos parecen ser la primera opción tanto en niños como en adultos ${ }^{7,8}$.

\section{BIBLIOGRAFÍA}

1. Picus D, Frank P. Eosinophilic esophagitis. Am J Roentgenol. 1981; 136:1001-3.

2. Attowd SE, Smyrk TC, Demeester TR, Jones JB. Esophageal eosinophilia with dysphagia. A distinct clinicopathologic syndrome. Dig Dis Sci. 1993; 38:109-16.

3. Fox V, Nurko S, Furuta G. Eosinophilia esophagitis: it's not just kid's stuff. Gastrointest Endosc. 2002; 56:260-70.
4. Argüelles Arias F, Sánchez-Gey Venegas S, Herrerías Gutierrez JM. Otras esofagitis. Etiología infecciosa. Etiología tóxica/caústica. Esofagitis eosinofílica. Medicine. 2008; 10(1):12-19.

5. Sojo Aguirre A. Esofagitis eosinofílica. Bol. S Vasco-Nav Pediatr. 2009; 41:2-8.

6. Straumann A, Simon HV. Eosinophilia esophagitis: escalating epidemiology? J Allergy Clin Inmunol. 2005; 115:418-19.

7. Lucendo Villarín AJ, De Rezende L. Esofagitis eosinofílica. Revisión de los conceptos fisiopatológicos y clínicos actuales. Gastroenterol Hepatol. 2007; 30(4):234-43.

8. Beltrán C, García R, Espino A, Silva C. Esofagitis eosinófila: una entidad emergente. Rev Otorrinolaringol Cir Cabeza cuello. 2009; 69:287-98.

9. Rojas Calderón I. Esofagitis eosinofílica en niños. Revista médica de Costa Rica y Centroamérica. 2009; LXVII(590): 383-87.

10. Martín de Carpi J, Gómez Chiari M, Castejón Ponce E, Masiques Mas ML, Vilar Escrigas P, Varea Calderón V. Aumento del diagnóstico de esofagitis eosinofílica en nuestro medio. An Pediatr (Barc). 2005; 62(4):333-9. 